\title{
Development f Smart Garbage Bins for Automated Segregation of Waste with Real- Time Monitoring using Iot
}

\author{
Rishabh Jain, Oindrila Halder, Pranit Sharma, Akshita Jain, E. Elamaran
}

\begin{abstract}
The Internet of Things (IoT), as an infrastructure planned for the proposed Smart City concept, offers new opportunities for urban service management. The IoT vision presents promising and cost-effective solutions for massive data collection and analysis, which can be applied to many areas, helping them to operate more efficiently. Normally, one of the most difficult problems in smart cities is the collection of urban waste. To optimize the waste collection logistic procedure, we provide a prototype focused on the field above. In this paper we are proposing a real time waste collection and management, through smart bins, in which the waste is first segregated and collected in dedicated bins which are monitored in real-time on the cloud. Whenever the garbage bins fill, we get an email notification as an alert which is sent to an authorized person. Also, an email alert is sent in case a fire is detected.
\end{abstract}

Index Terms-Internet of things, Waste collection, Cloud, Email alert, Smart bins

\section{INTRODUCTION}

The Internet of Things (IoT) is a vast area that has been emerging at a dizzying pace in recent years and has received much attention. The Internet of Things (IoT) can play an important role in making cities greener, safer and more efficient [1]. Improve cleaning and, consequently, quality of life can be achieved by connecting devices, vehicles and infrastructure throughout the city. The best technological solutions can be obtained in smart cities by separating waste into the same source, in order to facilitate the collection and separation of these.

A scheme of smart waste management using Wireless Sensor Networks (WSNs) and IoT (Internet of Things) is a feasible solution to the problem at hand $[1,2]$. This new framework for waste management system aims at bridging the gap between the data availability at the source and the availability of the same at the Municipal Corporation's end. Using this real-time data, the Municipal Corporation can

Revised Manuscript Received on August 14, 2019.

Rishabh Jain, Department of Electronics and Communication, SRM Institute of Science and Technology, Kattanulathur, Chennai, Tamilnadu, India.(email: rishabhmaster1 @gmail.com)

Oindrila Halder, Department of Electronics and Communication, SRM Institute of Science and Technology, Kattanulathur, Chennai, Tamilnadu, India.(email: oindrilahalder@gmail.com)

Pranit Sharma, Department of Electronics and Communication, SRM Institute of Science and Technology, Kattanulathur, Chennai, Tamilnadu, India.(email: pranit.sharma03@gmail.com)

Akshita Jain, Department of Electronics and Communication, SRM Institute of Science and Technology, Kattanulathur, Chennai, Tamilnadu, India.(email: akshitajain2212@gmail.com)

E. Elamaran, Department of Electronics and Communication, SRM Institute of Science and Technology, Kattanulathur, Chennai, Tamilnadu, India.(email: elamaran.e@ktr.srmuniv.ac.in) arrange for the pick-up and better management of waste collection.

A huge inflation has been registered in the municipal solid waste generation all over the world. This is due to a variety of factors. Various municipal garbage bins face issues such as overflowing, and emanation of pungent smells. These issues arise due to the inefficient and lack of timely collection, which to an extent, are due to the lack of available data pertaining to the real-time status of the bins.

The containers are implemented with sensors and are connected in a network via WSN. The sensors in the bins collect the data for each given interval. Once the threshold is reached, a request is sent to the recovery agent, present at the municipal office. This agent collects requests from all overflowing garbage bins and communicates via the IoT framework. A prototype of equipment has been developed for the proposed structure. The analysis of the proposed scheme offers better results in terms of waste management. Real-time updates are sent to the agent via e-mail to facilitate dynamic real-time responses.

The proposed architecture of the system includes the setting up of smart garbage bins at the sender side, which detect the garbage level. These sensors are connected to the microcontroller unit. A power supply is given to the microcontroller to operate these sensors. Waste is segregated into wet waste, dry waste and metallic waste actively. The proposed mechanism for waste segregation is the use of a conveyer belt; however, for demonstration purposes a motorized platform is used to display waste segregation. The waste is first segregated and collected in dedicated bins, which are monitored in real-time in the Cloud. The data collected is sent to the receiver side, where the user gets a notification on a mobile or a laptop whenever the bin fills up or a fire is detected. According the indication of the level or in case of a fire, further course of action can be planned once the notification e-mail is received. A control center hosts the web-server, the database and the email notification/alert system. The control center runs two systems that manage the two different activities, namely, the e-mail notification/alert system and the web-based monitoring system on Cloud.

The objective is to determine the filled up garbage bins and provides an indication to the GCV (vehicle for waste collection). Therefore, the advanced discharge method or the

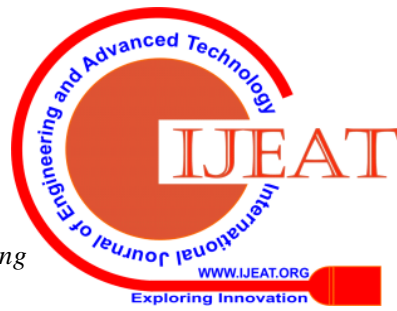



TIME MONITORING USING IOT

odours do not cause any inconvenience or discomfort.

Smart waste bins collect the status of the garbage bins and wirelessly transmits the data to municipal corporation office. Once the data is received at the municipal corporation office [6], it stores these values inside a database. E-mail notification/alert is automatically sent to the concerned authorities, updating the current status of the waste bins. As the components used are cheap, the development costs will be economical. For future works, the paper proposes the use of a customer complaints module and its integration with the e-mail notification/alerts system to trigger quick and dynamic responses.

The organization of this paper contains the following sections. Section 2 covers the related works; section 3 describes the Proposed System and Model. Methodology is highlighted in Section 4. Section 5 provides the Inference. Section 6 underlines the possible Future Works. Some limitations are mentioned in Section 7 and the Conclusion is covered in Section 8.

\section{RELATED WORKS}

In the past few years, advancements in the field of IoT and the use of smart sensors in various industries, has paved the way for the improvement and integration of the two with each other. A plethora of work has been done on the use of various sensors in multidisciplinary fields, which include, mapping them with IoT to come up with innovative solutions.

Development of smart cities [1] relies typically on the use of various sensors and most importantly on the use of IoT, to map and bridge the gap between them in order to develop a system that is self-sustaining and requires minimal input.

With the advancements in driverless cars $[10,11,12]$ taking place, the same can very well be integrated and utilized to ensure timely pick up and to leave out the need for any human intervention throughout the process.

Development and advancements in Cloud computing [2] plays an absolutely necessary role to ensure the smooth functioning and better latency of the system.

Data sent to the control system needs to be Control System needs to be stored and maintained in the Cloud, as well as in the Database, for which a data storage framework needs to be put in place [9]. This is to enable better analytics of the data collected over time. This leads to a better understanding of patterns in waste generation, so as to come up with precise predictions for resource allocation in the future.

\section{PROPOSED SYSTEM}

The prototype proposed is designed to increase the efficiency of waste segregation by implementing the concept of IoT and automation while keeping it cost effective and sustainable to use. The suggested prototype consists of an open source IoT platform based microcontroller called Node-MCU with an ESP8266 Wi-Fi module. An IR sensor is mounted to detect whenever an object is placed in the setup. A moisture sensor, TE215 Hygrometer Module, is interfaced with the Node-MCU to differentiate between wet and dry waste. A metal detector is further interfaced to detect metallic waste. Each category of waste has a dedicated bin to collect the segregated waste in it. Various mechanical approaches can be used to facilitate the segregation of waste out of which the approach of a tilting platform is used for the dumping of waste material into dedicated bins after triggering the required sensors. An ultrasonic sensor is placed in each bin to monitor the waste level in the bin thus preventing overflow of garbage. Each bin will also have a temperature sensor placed in it to detect fire in the bins. The ultrasonic and temperature sensors will continuously monitor the bins. Ultrasonic sensor sends an alert in the form of an email when the dustbin is full. Similarly when a fire is detected the temperature sensor sends an email alert. This helps in taking appropriate action effectively. The alert system utilizes the Wi-Fi module via which such alerts are sent. The alert from ultrasonic sensor is used to inform the respective garbage truck to empty the bin and take it to respective waste processing unit. The alerts from temperature sensor go to the nearest fire station so that immediate action to douse the fire can be taken.

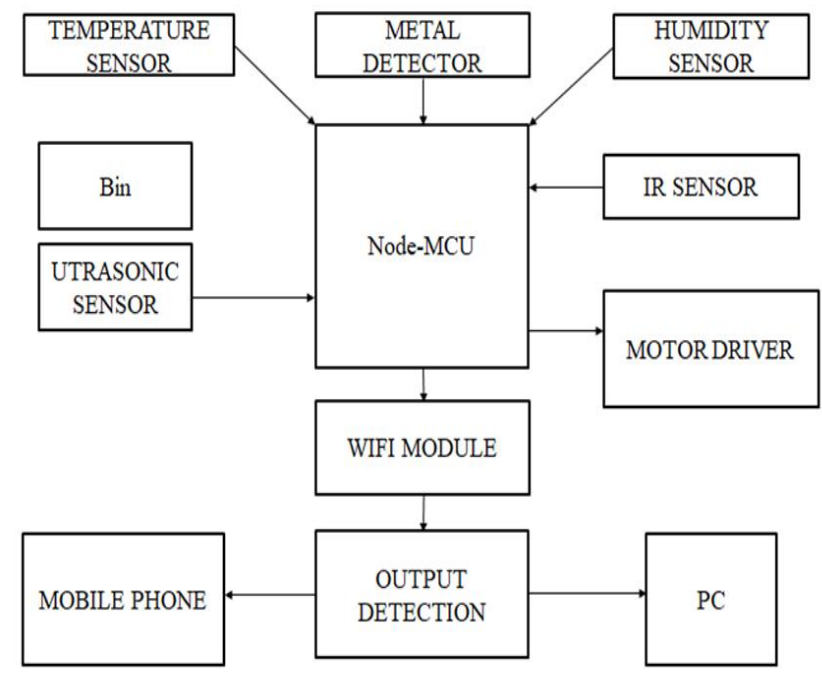

Fig. 1 Flow Diagram

\section{METHODOLOGY}

As soon as the waste material comes in contact with the IR sensor, it gets detected and is categorized as dry waste and is dumped into the dedicated bin for dry waste. If the waste material also triggers the moisture sensor along with the IR sensor, the detected waste is categorized as wet waste and is dumped into the dedicated bin for wet waste. Similarly, if the waste material triggers the metal detector along with the IR sensor, the detected waste is categorized as metallic waste and is dumped into the bin dedicated for metallic waste. Once the process runs continuously, the bins start to fill up over time. The ultrasonic sensors in each bin monitor the level of the waste in the bin which is monitored in real time on cloud service ThingSpeak. As soon as the threshold is crossed, an email alert is sent indicating that the garbage bin is full and needs to be emptied or replaced. The fire sensor is present in the bins and as soon as a temperature rise above $60 \mathrm{C}$ is detected, it sends an email 
alert indicating that a fire has been detected allowing for timely action to douse the fire. Thus, the possible spread of fire is stopped and the pollution due to the burning is prevented. As indicated in Fig. 7, the graphs show the level of the waste in the garbage bin at the time of testing. The two graphs belong to the two bins used while testing the working prototype of the project. The e-mail alerts sent during the testing are represented in Fig. 8. There is an email alert whenever the garbage bin is full and whenever the temperature sensor detects a temperature above $60 \mathrm{C}$ which has been set as the desired threshold.

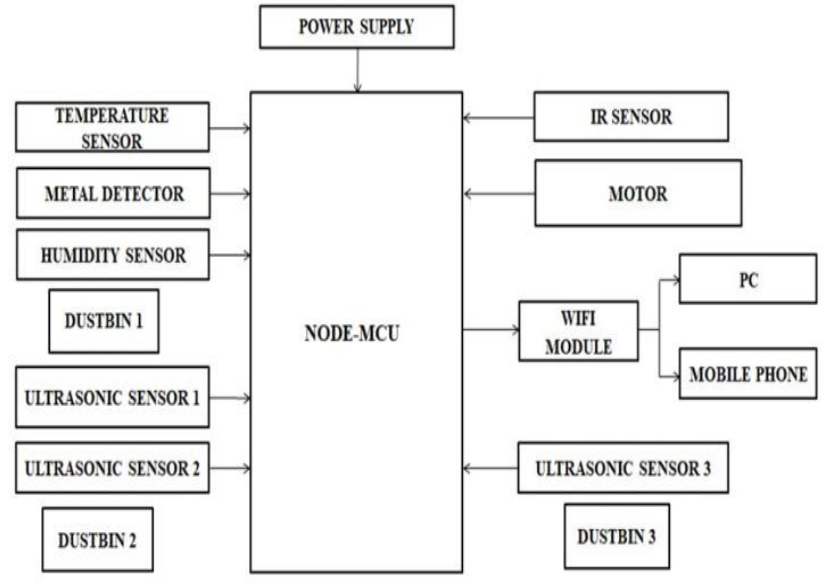

Fig. 2 Block Diagram

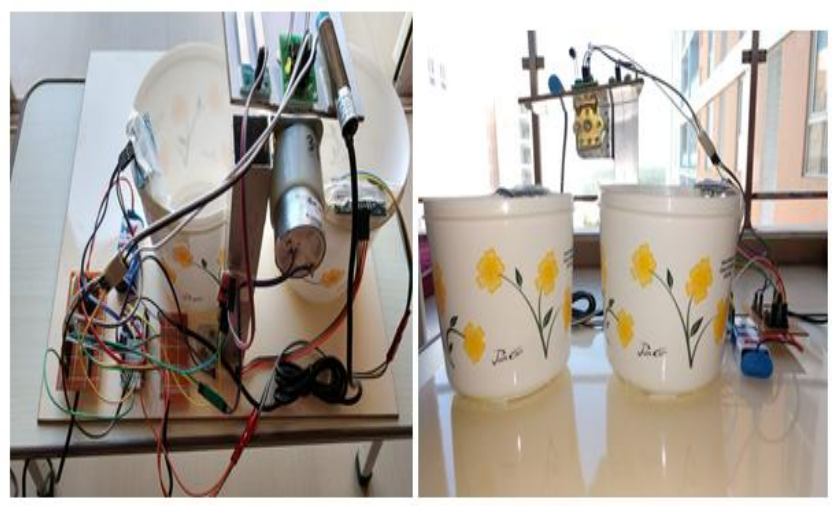

Fig. 3 Top View

Fig. 4 Front View

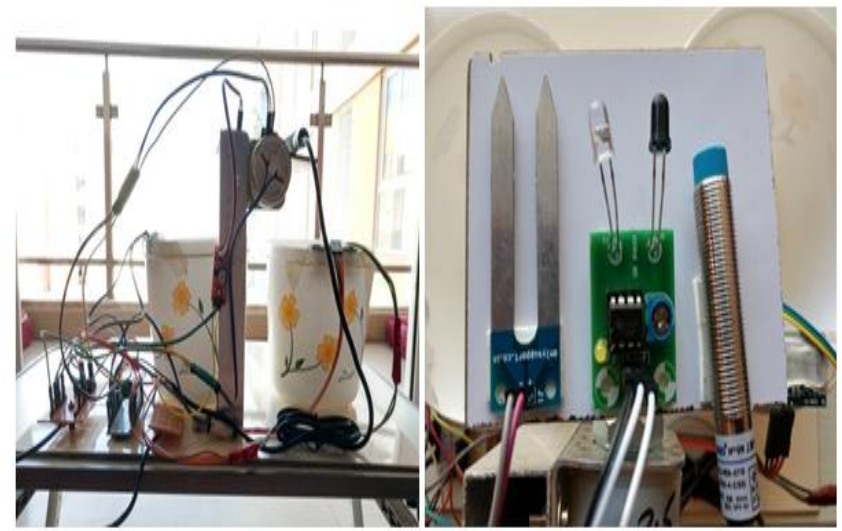

Fig. 5 Back View

Fig. 6 Sensors Used

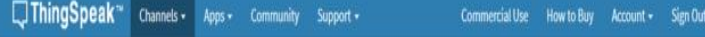

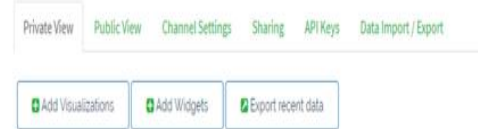

Channel Stats

Cratter: 4 dasage

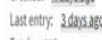

Enties 4.5

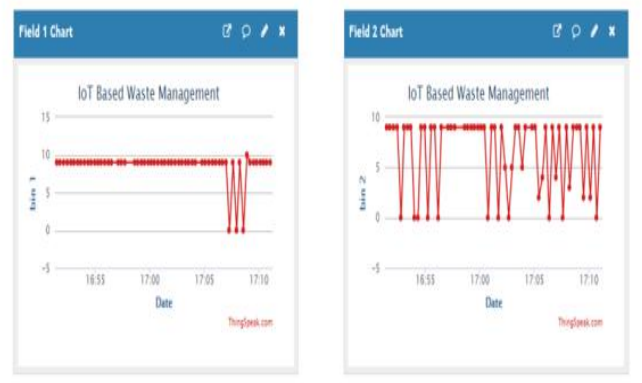

Fig. 7 Real-Time Status of Garbage Bins

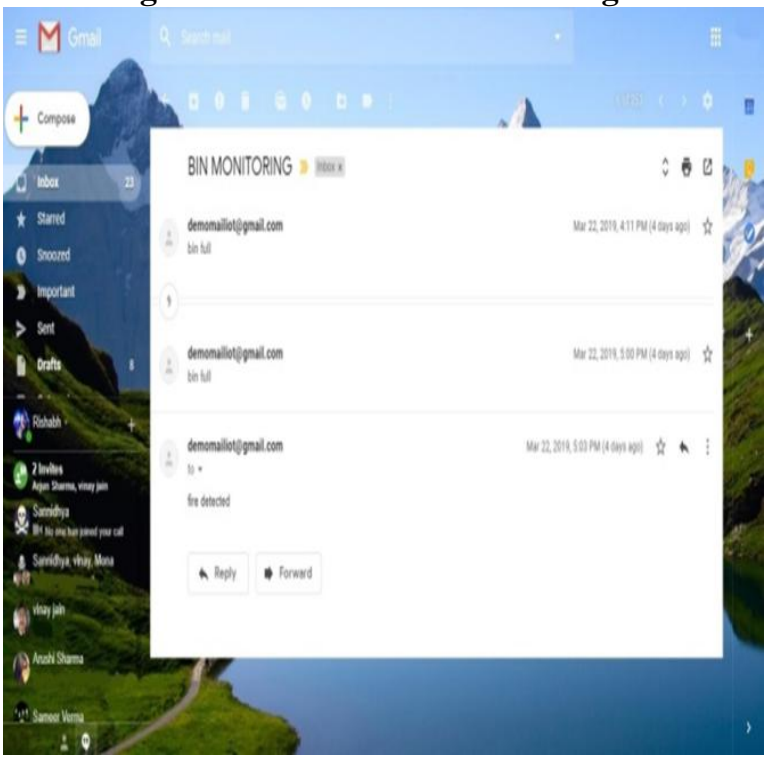

Fig. 8 E-mail Alerts

\section{INFERENCE \& RESULTS}

This project is very effective in managing waste in any big city. Rather than using conventional periodic collection method, priority-based system is used to clean the city without any overflowing dumpsters. It has been tested and verified properly to make sure all the different part work together for a smooth function of the whole system. The

proposed system provides efficient method to collect maximum waste while minimizing cost and maximizing efficiency. The system segregate waste into separate dedicated garbage bins and provide Store real time information about garbage bin status i.e. whether the bin is full or not. The system summarizes the collected information and generates the report and sends the email alerts.

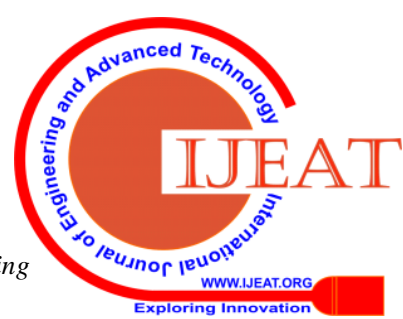




\section{FUTURE WORKS}

The future works include increasing the efficiency and eradicating the need for human intervention all throughout the process of waste collection. As the waste bins are equipped with sensors that send real-time information to the Control Centers, using the WiFi module, a pre-defined course of action can be set for various scenarios.

With the onset of driverless cars [12] and the advancements in route planning and optimization [11], the collection process can be integrated seamlessly with the real-time data, which is made available to the control system.

The advancements in these fields would mean lesser human intervention and in turn, lesser chances of error as well as faster response rates and better efficiency. With the eradication of the need for constant human monitoring, the The upfront cost of setup would be the only major cost associated with the system and thus, the efficiency and cost effectiveness of the system would be much better than that of the present system.

With the Internet of Things (IoT) offering seamless connectivity between various devices, systems and users, the users expect quick and on-the-go connectivity. These devices include smartphones, smartwatches, computers, laptops, etc. With quick and seamless connectivity, the integrity and security of the system becomes questionable. Since the proposed system is centralized and utilizes a common framework for an entire area, district or even a country, the integrity and data security of the same would be of utmost importance, so as to ensure uninterrupted and ontime operation of the system. A user access control can be defined at the end-points so as to minimize chances of replay, man-in-the-middle and denial of service attacks on the system [8]. In order to tackle the inherent security flaws that may present themselves during the course of the implementation of the system, cryptography, signal/image processing and communication capabilities for authorization and authentication functions, as compared to traditional approaches is proposed.

The efficient and reliable delivery of data-aware services is very closely related to the future of smart cities. We can personalize IoT systems, to suit the needs of the end user. Proposed future work includes leveraging a novel approach under the fog architecture [13] in order to bring connectivity and processing potential of the framework closer to the user, and improving the coupling of services with local IoT components in user-centric contexts. The possible gain in the access latency and context-sensitive service matching will enable a large number of smart city applications.

\section{LIMITATIONS/CONSTRAINTS}

The constant need for internet connectivity in order to make the data readily available to the control system might be a challenge in countries where data on-the-go services are not very established and thus, may add to the initial setup cost of the system.

The lack of a proper security framework and the strengthening of the security protocols of the system is necessary for the implementation so as to ensure the data costs of upkeep and regular maintenance will be minimal.

sent from either end is secure and reaches the intended receiver only.

The minimal, though existent need for human intervention for constant monitoring, may lead to a decreased efficiency and may leave a scope for error. Also, the costs involved with systems requiring human intervention are generally much higher than those associated with a completely automated system. This is because the costs associated with human-involving systems involve constant and recurring costs such as wages, which need to be paid.

\section{CONCLUSION}

With the help of a practical circuit implementation the procedure of waste collection is optimized. The system proposed in this paper provides an efficient method to collect and segregate waste, while minimizing overall cost and maximizing efficiency. This system segregates the waste into separate dedicated garbage bins and provides real time information about the garbage bin status, i.e., whether the bin is full or not and if there is a fire. The system summarizes the information collected, generates reports and sends alerts via e-mail.

\section{REFERENCES}

1. Andrea Zanella, Nicola Bui, Angelo Castellani, Lorenzo Vangelista, Michelle Zorzi: "Internet of Things for Smart Cities" IEEE Internet of Things Journal, Vol. 1, Issue: 1, Feb. 2014

2. Bin Cheng, Gürkan Solmaz, Flavio Cirillo, Ernö Kovacs, Kazuyuki Terasawa, Atsushi Kitazawa, "FogFlow: Easy Programming of IoT Services Over Cloud and Edges for Smart Cities", IEEE, Internet of Things Journal, Vol. 5, Issue:2, April 2018

3. Sam Aleyadeh: An IoT based architecture for waste management", IEEE, Vol. 2018, No.3, pp. 113-120, 2018

4. Keita Matsuo, Yuichi Ogata, Kouhei Umezaki, Evjola Spaho: "Design and Implementation of Waste Management Robots", IPSJ Journal, Vol. 2012, CVIM170, No.41, pp. 1-6, 2012

5. Sivasankari, Bhanu Shri, Y.Bevish Jinila: "Smart waste management using WSN and IoT", IEEE Vol. 2, No.4, pp. 211-227, 2017

6. Fachmin folianto, Yong Sheng low, Wai Leong Yeow: "Smart bins: smart waste management system", Journal of Telecommunication Systems, Springer, Vol. 38, No. 3-4, pp. 133-146, 2016

7. Norfadzlia Mohd. Yusof, Mohd. Faizal Zulkifli, Nor Yusma Anira Mohd. Yusof,Azziana Afififie Azman: "Smart waste bin with real time monitoring system", IEEE, Vol. 2018, No.3, pp. 113-120, 2018

8. Michael W. Condry ; Catherine Blackadar Nelson: "Using Smart Edge IoT Devices for Safer, Rapid Response With Industry IoT Control Operations", IEEE, Proceedings of the IEEE, Vol. 104, Issue: 5, May 2016

9. Lihong Jiang ; Li Da Xu ; Hongming Cai ; Zuhai Jiang ; Fenglin Bu ; Boyi Xu: "An IoT-Oriented Data Storage Framework in Cloud Computing Platform, IEEE, Transactions on Industrial Informatics, Vol. 10, Issue: 2, May 2014

10. László CSURGAI-HORVÁTH ; János Z. BITÓ: "Route Planning for Mobile IoT Devices", IEEE, 11th 
International Symposium on Communication Systems, Networks and Digital Processing (CSNDSP)

11. Lingli Yu ; Decheng Kong ; Xuanya Shao ; Xiaoxin Yan: "A Path Planning and Navigation Control System Design for Driverless Electric Bus", IEEE Access, Vol. 6

12. Brian Markwalter: "The Path to Driverless Cars (CTA Insights)", IEEE Consumer Electronics Magazine, Vol. 6, Issue: 2, April 2017

13. Sharief M. A. Oteafy; Hossam S. Hassanein: "IoT in the Fog: A Roadmap for Data-Centric IoT Development", IEEE Communications Magazine, Vol. 56, Issue: 3, March 2018 